[Berliner entomologische Zeitschrift 1874.]

\title{
Wieviel und welche Asida-Arten besitzen wir in Deutschland und der Schweiz?
}

\author{
Von
}

Dr. G. Kraatz.

I

ch habe in meinem Verzeichnisse der Käfer Deutschlands z w ei Asida-Arten aufgezählt, nämlich die früher hauptsächlich unter dem Namen grisea Ol. verbreitete, und die fascicularis Germ., in welcher bisweilen auch eine deutsche Art vermuthet wurde. Nun hat uns aber Herr Allard in seiner Monographie ') der Gattung Asida mit zwei neuen, deutschen Arten beschenkt, d. h. es wird von ihm Asida morbillosa Duftschmid von Triest als eigene deutsche Art aufgeführt und beschrieben (a. a. O. p. 178), und ebenso helvetica Sol. (a. a. O. p. 176), welche nach ihm bei Triest und in Italien vorkommen soll.

Auffallend ist, dals Redtenbacher (Faun. austr. ed. IIl. p. 87) von einer österreichischen oder Triestiner Asida gar nichts erwähnt, sondern nur sagt: "eine der gemeinsten Arten ist Asida grisea Fabr., kommt nach v. Heyden's Angabe zwischen Bingen und Bonn am Laacher See vor". Allard kennt nun eine grisea Fabr. ${ }^{2}$ ) gar nicht, sondern nur eine grisea $\mathrm{Ol}$, welche jedoch bei ihm unter rugosa Fourcroy (Ent. 1785. I. 157. 2.) zu suchen ist. In der That hätten wir uns des älteren Fourcroy'schen Namens wohl zu bedienen, hätte nicht Goeze (Entom. Beitr. I. pag. 687) bereits 1777 den im Jahre 1772 in Geoffroy's Ins. Paris. I. 347. 2. pl. 6. f. 6 . beschriebenen und abgebildeten, aber nicht benannten Tenebrion ridé etc. Tenebrio sabulosus benannt; wir werden hierauf durch den Gemminger-Harold'schen Catalog p. 1878 ganz richtig hingeführt, und es ist H. v. Harold's Verdienst zuerst auf die Goeze'sche Benennung aufmerksam gemacht zu haben; es mag daher von nun ab überall der Name sabulosa Goeze statt rugosa Fourcr. (= grisea Ol.) gebraucht werden.

') in de Marseul's Abeille VI. p. 159-304.

${ }^{2}$ ) Vergl. über grisea Fabr. das unter dieser Ueberschrift Gesagte. 


\section{Asida grisea Fabr. (non Ol.).}

Als ältester Autor einer Asida grisea ist Olivier bei Allard angegeben, grisea Fabr. finde ich bei ihm nicht eitirt; im Gemminger-Harold'schen Cataloge ist Fabricius als der älteste Autor von 1781 vor Olivier citirt. Allard's Vernachlässigung des Autors Fabricius ist um so weniger gerechtfertigt, als bereits Mulsant Latigènes 1854. pag. 91 unter Asida grisea ausdrücklich hervorhebt, dafs Opatrum griseum Fabr. "sans aucun doute" vom Opatrum griseum Ol. verschieden sei; einer Bemerkung der im Gemminger-Harold'schen Catalog noch nicht Rechnung getragen ist. Hat Mulsant, der sich auf die Beschreibung stützt, Recht? oder eine alte Tradition, die die As. rugosa Fourcr. All. (= grisea Ol.) auf grisea Fabr. deutet? Schaum's kritischer Catal. Col. Eur. führt auch eine grisea Ol., nicht Fabr. auf. - Fabricius giebt als Vaterland seiner grisea (Spec. Ins. I. pag. 89) Italien an; Mulsant deutet sie fraglich auf Dejeanii, welche Allard indessen nur aus dem südlichen Frankreich kennt. Meines Erachtens ist aber die Vaterlandsangabe Italien bei Fabricius mit um so grölserem Gewichte zu belegen, je kürzer seine Beschreibung ist. Der alte italiaenische Autor, der ohne Bedenken eine italiaenische Asida als Opatrum griseum Fabr. bereits 1795 aufführt, aber weder von Mulsant, noch Gemminger, noch Allard citirt wird, ist Rossi, und zwar giebt derselbe als Fundort seiner grisea Florenz an. Nun bemerkt Allard ausdrücklich, dafs Bellier de la Chavignerie die bis nach Corsica verbreitete longicollis Sol. All. bei dieser Stadt aufgefunden habe. Hiernach scheint mir die einfachste und ungezwungenste Deutung für As. grisea Fabr. die auf longicollis Sol. zu sein.

Es wäre doch die erste Pflicht der früheren Autoren gewesen, sich zu fragen, welche italiaenische Asida wobl auf die italia en is che grisea Fabr. gedeutet werden könne, und Rossi's Fauna etrusca (ed. Hellw. I. p. 60) zur Hand zu nehmen. Statt dessen bekümmert der eine sich um die grisea Fabr. gar nicht, der andere deutet eine französische Art auf dieselbe, und schliefslich folgt vielleicht wieder eine Tirade von H. de Marseul, der Allard's Monographie zum Druck gebracht hat, dafs derjenige, der As. longicollis Sol. All. zu grisea Fabr. zieht, kein vrai entomologiste sondern bouquinier sei, weil er Rossi's Fauna zu Rath gezogen hat.

\section{Asida morbillosa Fabr.}

wurde bisher mit Recht als synonym von grisea Ol. aufgeführt, und zwar zieht Fabricius selbst (Syst. El. I. pag. 140) zu seiner 
morbillosa seine variolosa Fabr. Ent. Syst. Suppl. 45. 24. und die variolosa Faun. Germ. ohne näheres Citat. Als Vaterland der morbillosa Fabr. und variolosa Fabr. wird beide Male angegeben: Habitat in Germania australiori Dom. Panzer.

Natürlich ist unter diesen Umständen der Vergleich von Panz er Faun. Ins. Germ. Heft 74. No. 1. nebst Abbildung wichtig; der daselbst abgebildete Käfer ist offenbar ein männliches, schwach (vielleicht unwillkürlich) vergröfsertes Ex. der sabulosa, da es über 6 Linien Länge hat. Panzer sagt ausdrücklich, dafs er vor mehreren Jahren an Fabricius 1 Exemplar mitgetheilt habe, welches er als Silpha rugosa Scopoli 53 erhalten und Fabricius sogleich für seine Pim. variolosa erklärt hatte.

Was thut nun der Monograph Allard? er citirt p. 178 die morbillosa Fabr. fraglich unter der morbillosa Duft. und ohne irgend welches Fragezeichen p. 1871 unter fascicularis ') Germ.; trotz dieser bestimmten Vereinigung der süddeutschen morbillosa Fabr. mit der fascicularis sagt er am Schlufs der letzteren: cette espèce paraît propre à la Dalmatie.

In Folge dieser bestimmten Vereinigung der morbillosa Fabr. mit der fascicularis wird im Gemminger-Harold'schen Cataloge p. 1877 die fascicularis Germ. als Synonym un ter morbillosa Fabr. gesetzt ${ }^{2}$ ) und als Vaterland der morbillosa Fabr. fälschlich Dalmatien angegeben.

Weiter wird der Name der morbillosa Duftsch., welche Allard als eigene Art festhält, p. 1875 in Duftschmidi Gemminger umgeändert, weil natürlich nicht eine morbillos $a$ Duft. neben einer mor-

') Hierin hat er wahrscheinlich Recht, obwohl er mir ein Schweizer Ex. meiner Sammlung (No. 41. bezettelt) als fascicularis bestimmte. Es scheint mir nicht unpraktisch bei der Besprechung solcher Stücke, bei denen meine Bestimmung von der des Monogiaphen abweicht, die Nummer in Parenthese hinzuzufügen, unter welcher sie der Monograph von mir erhalten hat; diese No., welche fast immer roth sind, verbleiben stets an den Nadeln und ermöglichen jederzeit den Vergleich mit der Benennung auf der Original-Liste, welche so lange als möglich aufbewahrt bleibt. Ich würde Aehnliches auch den Besitzern anderer Sammlungen e:npfehlen, namentlich die Einführung von No. derselben, Farbe und Gröfse für alle an verschiedene Monographen mitgetheilten Stücke. Erhält der Monograph mehrere Stücke von derselben Art oder demselben Fundorte etc., so erhält nur eins eine rothe No., die anderen ein kleines, viereckiges Papier von derselben Farbe.

${ }^{2}$ ) Was Allard inconsequenter Weise unterläfst. 
billosa Fabr. festgehalten werden kann, und durch die hinzugefügte Vaterlandsangabe Hungaria wird die echt deutsche Duftschmid'sche Art nach Ungarn versetzt. ${ }^{1}$ )

So bringen Allard's Flüchtigkeiten und Inconsequenzen neue Irrthümer zu Wege.

\section{Asida Duftschmidi Gemminger} in v. Harold's Col. Hefte VI. p. 222.

ㅇ As. morbillosa Duft. Faun. austr. II. p. 290. -- All ard Revis. p. 178.

б' As. variolosa Duft. Faun. austr. II. p. 291.

Das Hauptmerkmal dieser angeblichen Art besteht in der forme manifestement plus large et plus courte und der Grölse (ð 12 mill., Q 13-15 mill.).

Erwägen wir, dafs die nach allen bisherigen Annahmen weit verbreitete sabulosa an verschiedenen Localitäten verschieden gut gedeihen mufs, wie bereits Gredler (unter seiner morbillosa Fabr.) hervorhebt, dafs aber in Illyrien etc. auch z. B. Carabus irregularis, violaceus als Germari, convexus als dilatatus etc. besonders gut gedeihen, so mufs es uns viel natürlicher erscheinen, dafs auf dem Karst und bei Triest eine besonders kräftige, gedrungene Raçe der sabulosa auftritt, als dafs die dort vorkommenden grofsen Expl. eine eigene Art zu bilden haben. Und neben dieser Art soll bei Triest noch die helvetica vorkommen! und warum bleibt die über ganz Frankreich bis nach Sicilien verbreitete sabulosa von der Triestiner Küste fort?

Allard giebt an (p. 179), dafs die Duftschmidi nach v. Heyden in den Apenninen und Italien vorkomme.

Neben Asida morbillosa wird von Duftschmid eine Asida variolosa, auf welche er die variolosa Fabr. bezieht, festgehalten; auch diese stammt von Triest, ist aber nur 5 (statt 6) Linien lang, schmaler, gleichbreit, der Hinterleib viel minder gewölbt, die Deckschilde gegen hinten nicht eingedrückt etc. Meines Erachtens haben wir in derselben unzweifelhaft das Männchen der morbillosa Duft. zu erkennen.

Allard ignorirt diese variolosa Duft.

$\left.{ }^{1}\right)$ In ähnlichen Fällen wo eine von mehreren Vaterlandsangaben zu einer Art gesetzt wird, scheint es mir nothwendig, immer die Lokalität in erster Linie oder allein anzugeben, aus welcher das vom Autor beschriebene Ex. stammt. 
4. Asida helvetica Sol. Annal. Soc. Ent. France 1836. p. 418. - Allard Revis. p. 176. 3.

Diese Art ist von Solier nach ein em Ex., von Allard nach einigen Paaren aus der Schweiz aufgestellt und kommt nach Letzterem auch bei Triest und in Italien vor. Stierlin (Faun. Col. Helv. p. 217) giebt als einzigen Fundort der helvetica an: Fufs des Salève (Tournier). Dagegen nennt er die sabulosa (grisea) im Waadt und Wallis nicht selten, und giebt Genf, den Jura und Reculet (Bonvouloir) als weitere Fundorte an. Da nun Allard seine Schweizer Expl. von de Bonvouloir erhielt, so sind höchst wahrscheinlich die Bonvouloir'schen Schweizer Stücke bei Stierlin sabulosa, bei Allard helvetica.

Mir selbst bestimmte Allard ein Stück von Trient als helvetica, ferner ein sehr davon abweichendes, besonders dunkles, italiaenisches; über dieses und ein zweites ähnliches von derselben Localität will ich hier kein bestimmtes Urtheil abgeben, dagegen spreche ich meine vier Trientiner Stücke mit Bestimmtheit als kleine sabulosa an; die 3 옹 sind noch etwas kleiner und kürzer als die $\&$ der französischen sabulosa in der Regel sind.

$\mathrm{Zu}$ beachten ist, dafs helvetica nach Allard auch bei Triest vorkommt, wo unzweifelhaft die Duftschmidi nicht fehlt und nach gewöhnlicher Bestimmung die rugosa All. (grisea) vorkommt, die Allard auf Frankreich und das südliche Sicilien beschränkt.

Die Triestiner Entomologen mögen nun auch ein Wort mitsprechen, ob 3, 2 oder 1 Art nach ihrer Ansicht dort vorkommt, abgesehen von der Mögli chkeit des Vorkommens der fascicularis.

Aber auch die Tyroler haben nach Allard's Determination ein Wort über helvetica mitzusprechen. Gredler führt von der einzigen von ihm angenommenen, als grisea Ol. (morbillosa Fabr.) aufgeführten Art eine Menge von Fundorten an, wobei hervorzuheben ist, dafs seine grisea in den Weinbergen der Ebene klein und selten ist.

Es ist merkwürdig, dafs H. Allard bei allen seinen Angaben über die verschiedene Grölse und Gestalt der von ihm neben sabulosa angenommenen Arten sich so wenig Mühe giebt uns constante greifbare Merkmale zu ihrer Unterscheidung zu geben; wer nur ein wenig die Variabilität der Asida kennt, und die sollte H. Allard doch kennen, der verzweifelt entweder an der Möglichlichkeit zu bestimmen, oder er bestimmt falsch oder gar nicht; der letztere Weg scheint mir der praktischste, mit andern Worten: der 
Eindruck der meisten Beschreibungen der hier besprochenen Arten ist ein so ungünstiger, dafs es mir verlorene Zeit und Mühe scheint, die specifische Stichhaltigkeit oder die Hinfälligkeit der vielen un peu plus et un peu moins kritisch nachzuprüfen Um so empfänglicher würde ich für Belehrung von anderer Seite sein und den Vorwurf der Flüchtigkeit von Allard zurück und auf mich nehmen, wenn man uns Duftschmidi und helvetica als gute Arten kennen lebrt.

Ueber einige der sabulosa nahe verwandte Arten will ich hier noch einige Worte hinzufügen.

\section{Asida lutosa Sol.}

Allard bestimmte mir ein Ex. der lutosa (No. 11. roth bezettelt) trotz der ausdrücklichen Namensangabe und Vaterlandsbezeichnung Krimm als fascicularis $\$$; es scheint mir nicht unwichtig dies hervorzuheben, damit eventuell Andere entscheiden können, ob etwa wirklich fascicularis in der Krimm vorkommt; Alles was ich von dort besitze ( $7 \mathrm{Ex}$. von Ribbe gesammelt, 2 von Frivaldszky aus "Süd-Rufsland", dazu eins von Kretschmar jun. aus dem Caucasus) halte ich für lutosa.

\section{Asida obesa (Chevr. in Coll.) Allard Rev. p. 179.}

Ich will mir kein bestimmtes Urtheil über die Art aus Algier erlauben, da ich sie von dort nicht besitze; ein mir von Allard als obesa bestimmtes (6. roth bezettelt) männliches Ex. von Constantinopel (Frivaldszky) und ein Pärchen von derselben Lokalität, wage ich nicht von der sabulosa zu trennen.

Für die Güte der Art spricht der Umstand, dafs sie in Chevrolat's Sammlung zu einer solchen gestempelt war, gerade nicht besonders; auch ist das von Allard in der Einleitung der Beschreibung hervorgehobene Merkmal: "En outre elle a une teinte ferrugineuse qu'elle doit à la terre de cette couleur qui la recouvre ordinairement" ebensowohl für die Güte der Beschreibung, als den Monographen der Asida-Arten charakteristisch!

\section{Asida catenulata Muls. Latigènes. 1854. p. 92. -} Allard Rev. p. 175.

ist von Mulsant nach einem einzelnen $\sigma^{\top}$ aus der Lozère beschrieben, welches er anzweifelhaft für eine gute Art hält, welche indessen rêclame des nouvelles observations etc. Allard erbielt den Käfer von H. Marquet, der mehrere Ex. aus dem südl. Frankreich, 
Spanien und Algier besitzt. Mir bestimmte Allard ein Stück (No. 4 . roth bezettelt) aus Toscana (vom Bruck) und ein grofses (No. 1. roth bezettelt) aus der Schaum'schen Sammlung mit der Vaterlandsangabe Spanien als catenulata. Beide würde ich nicht von sabulosa zu trennen wagen.

Wirkliche oder angebliche Stücke der catenulata, womöglich mit Hervorhebung der charakteristischen Merkmale, würden mir von grossem Interesse sein.

8. Asida fascicularis Germar Reise nach Dalmat. 1817. p. 159. No.53. (Allard citirt p. 53.)

Unter Asida morbillosa Fabr. ist ausgeführt, dafs die Germarsche Ari nicht zu derselben zu ziehen sei; allerdings citirt Germar selbst den Platynotus morbillosus Fabr. Duft. unter seinem Platynotus fascicularis, allein Autoren, die das alte Citat nicht einfach nachgeschrieben haben, wie Allard, sondern nachprüften, wie z. B. Mulsant, haben ganz richtig As. morbillosa zur sabulosa gezogen.

As. fascicularis ist zwar als deutsche Art angesprochen worden, doch hat man so wenig bestimmte Nachrichten über ihr Vorkommen in Deutschland, dafs es mir gegenwärtig am angemessensten erscheint, sie ganz aus der Reihe der deutschen Arten zu streichen. Wahrscheinlich hat man schon kleine Stücke der sabulosa auf sie bezogen, sie ist aber merklich kleiner als die kleinsten, von hellerer Grundfarbe, die Fühler feiner, heller röthlich braun, der Seitenrand des Halsschildes breiter abgesetzt, an den Seiten stärker gerundet.

Von Germar nach Ex. bei Zara beschrieben, die gegenwärtig in meinem Besitze sind.

Bei Zara kommt auch die, mit der fascicularis öfters verwechselte lineatocollis Küst. All. vor, welche sich von der ersteren leicht durch die scharfen, nach hinten vorgezogenen Hinterecken des Halsschildes unterscheidet, und namentlich von $\mathrm{Kahr}$ versendet wurde.

Aus alle dem ergiebt sich also, dafs wir bis jetzt nicht vier, sondern nur eine deutsche Asida kennen, welche vom südlichen Oesterreich und dem Karst westlich bis zum Laacher See vordringt. Ihre Nomenclatur wäre kurz folgende: 
sabulosa Goeze, Harold, Kraatz. Gallia

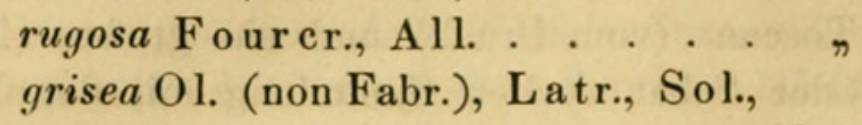

var. major Duftschmidi Gemming. . Germ. mer.

우 morbillosa Fabr., Duft., All. . " "

б’ variolosa Fabr., Duft., Panz. . " "

var. helvetica Sol., All. . . . . . Germ. m., Helvet., Ital.

var. glabricosta Sol. . . . . . Gall. m., Sicil.

var. insidiosa Muls. . . . . Gall. m. alp.

var.? obesa All. . . . . . . . . Alger.

grisea Fabr. Rossi. Krtz.

longicollis Sol. All.

\section{Asida pusillima, eine neue Art aus der Sierra Nevada \\ beschrieben von \\ Dr. G. Kra a $t z$.}

\section{Asida pusillima.}

Oblonga, deplanata, piceo-brunnea, opaca, subtiliter granulata, dense griseo-pubescens, terrulenta, antennis rufescentibus, breviusculis, articulis 4-9 latitudine parum longioribus, decimo transverso, capite thoraceque dense subtilissime punctato-granulatis, hoc lateribus valde reflexis, antice profunde, postice leviter et late emarginato, angulis posterioribus parum productis, acutiusculis, lobo medio perparum producto, elytris lateribus acute carinatis, planiusculis, costa sola laterali perspicua, vix interrupta, dorsali vix ulla, pedibus rufescentibus. - Long. $7-8$ mill., $3-3 \frac{1}{2}$ lin.

Fem.: Elytris posterius magis dilatatis, supra convexiusculis.

$2 \delta^{\sigma}, 1$ von mir in der Sierra Nevada unter Steinen gesammelt. 


\section{$2 \mathrm{BHL}$ Biodiversity Heritage Library}

Kraatz, G. 1874. "Wieviel und welche AsidaDArten besitzen wir in Deutschland und der Schweiz?" Berliner entomologische Zeitschrift / herausgegeben von dem Entomologischen Vereine in Berlin 18(102),105-112.

https://doi.org/10.1002/mmnd.18740180114.

View This Item Online: https://www.biodiversitylibrary.org/item/36410

DOI: https://doi.org/10.1002/mmnd.18740180114

Permalink: https://www.biodiversitylibrary.org/partpdf/209980

\section{Holding Institution}

Smithsonian Libraries

\section{Sponsored by}

Smithsonian

\section{Copyright \& Reuse}

Copyright Status: Public domain. The BHL considers that this work is no longer under copyright protection.

This document was created from content at the Biodiversity Heritage Library, the world's largest open access digital library for biodiversity literature and archives. Visit BHL at https://www.biodiversitylibrary.org. 